\title{
On the Correlated Weibull Fading Model and Its Applications
}

\author{
Nikos C. Sagias*, George K. Karagiannidis ${ }^{\dagger}$, Petros S. Bithas*, and P. Takis Mathiopoulos* \\ * Institute for Space Applications and Remote Sensing, National Observatory of Athens, \\ Metaxa \& Vas. Pavlou Street, 15236 Athens, Greece, E-mail: nsagias@space.noa.gr, pbithas@space.noa.gr, mathio@space.noa.gr \\ ${ }^{\dagger}$ Division of Telecommunications, Electrical and Computer Engineering Department, \\ Aristotle University of Thessaloniki, 54124 Thessaloniki, Greece, Email: geokarag@auth.gr
}

\begin{abstract}
Ascertaining on the suitability of the Weibull model to describe fading channels, a theoretical framework for the multivariate Weibull distribution, generated from correlated Gaussian elements, is presented. Concerning the bivariate Weibull model with arbitrary average fading powers, novel closed-form expressions for the joint probability density function (pdf), moments-generating function, cumulative distribution function (cdf), product moments, and the correlation coefficient are presented. Moreover, useful analytical formulae for the pdf and cdf of the multivariate Weibull distribution, with identical average fading powers and exponential correlation, are obtained. The derived theoretical results are applied to analytically evaluate the outage probability of selection diversity receivers, operating over correlated Weibull fading channels.
\end{abstract}

\section{INTRODUCTION}

Multivariate statistical analysis plays an important role for the investigation of wireless telecommunications system's performance in multi-channel fading environments, when correlation among diversity branches is assumed. This situation is applicable with diversity receivers in which antennae are not sufficiently separated, such as in wireless terminals with insufficient antenna spacing equipped with space and polarization antenna diversity (mobile terminal, indoor base-station, etc). In these applications, the fading among the channels is correlated, resulting in a degradation of the diversity gain obtained [1].

There are a few approaches to the multivariate analysis of fading models in the open technical literature. Nakagami in [2], has defined the $m$-bivariate probability density function (pdf), while Tan and Beaulieu [3] have derived an infinite series representation for the bivariate Nakagami- $m$ and Rayleigh cumulative distribution function (cdf). Alternatively, Simon and Alouini [4] have proposed an expression for the bivariate Rayleigh cdf in the form of a single integral with finite limits with the integrand composed of elementary functions. More recently, Karagiannidis et al. [5] have presented a formula for the multivariate Nakagami- $m$ pdf with exponential correlation and an infinite series approach for the corresponding cdf, bounding the error resulting from the truncation of the infinite series. The same authors have extended their analysis to the arbitrary correlated case, approximating the correlation matrix with a Green's matrix [6]. The theoretical results presented in [5], have been also used to study the performance of triplebranch [7] selection combining (SC) receivers over correlated Nakagami- $m$ fading channels. Finally, Mallik [8] has pre- sented useful theoretical results for the multivariate Rayleigh distribution, which are in agreement with those in [5]. In that work, the theoretical results have been used to the performance analysis of digital modulations over correlated Rayleigh fading channels with diversity combining. The Weibull distribution plays an important role in several scientific fields, such as in radar clutter, reliability engineering, and failure data analysis. Concerning wireless communications theory, the Weibull model exhibits an excellent fit to experimental fading channel measurements, for both indoor [9] and outdoor [10] environments. A physical justification for modelling wireless fading channels with the Weibull distribution has been given in [11]. However, only very recently, the topic of communications over Weibull fading channels begun to receive renewed interest. Considering the performance of diversity receivers over Weibull fading channels, a study for the evaluation of the generalized-selection combining (GSC) receiver's performance over independent Weibull fading channels has been presented [12]. More recently, three other contributions dealing with diversity combining, in independent [13], [14] and correlated [15] fading, have been presented. However, to the best of the authors' knowledge, there is not any published work in the open technical literature concerning correlative Weibull fading, generated from correlated Gaussian elements, and including applications on wireless communications theory.

In this paper, we present both the statistical analysis for the multivariate Weibull distribution and its applications in wireless communications systems. More specifically, the bivariate Weibull pdf, with arbitrary fading powers, is presented as a function of the Gaussian elements of the multipath components and closed-form expressions for the corresponding momentsgenerating function (mgf), cdf, product moments, and correlation coefficient are derived. The multivariate Weibull fading model, derived also from Gaussian elements, with exponential correlation and identical average fading powers is also investigated and useful analytical expressions for the joint pdf and cdf are presented. These novel theoretical results are applied to $\mathrm{SC}$ receivers, operating in correlated Weibull fading, where the outage probability is analytically derived.

\section{The Weibull Fading Model}

The fading model for the Weibull distribution considers a multipath wave propagating in a non-homogeneous environ- 


$$
\begin{aligned}
F_{R_{1}, R_{2}}\left(r_{1}, r_{2}\right)= & -\exp \left(-\frac{r_{1}^{2}}{\Omega_{1}}\right) Q_{1}\left(\sqrt{\frac{2}{1-\rho}} \frac{r_{2}}{\sqrt{\Omega_{2}}}, \sqrt{\frac{2 \rho}{1-\rho}} \frac{r_{1}}{\sqrt{\Omega_{1}}}\right) \\
& -\exp \left(-\frac{r_{2}^{2}}{\Omega_{2}}\right)\left[1-Q_{1}\left(\sqrt{\frac{2 \rho}{1-\rho}} \frac{r_{2}}{\sqrt{\Omega_{2}}}, \sqrt{\frac{2}{1-\rho}} \frac{r_{1}}{\sqrt{\Omega_{1}}}\right)\right]
\end{aligned}
$$

ment. At a given point the received signal encompasses a multipath component and the propagation environment is such that the resulting signal is observed as a non-linear function of the modulus of this component. Supposing also, that such a non-linearity is in the form of a power, the resulting envelope is observed as the modulus of the multipath component to the power of $2 / \beta>0$ [11].

Let's assume a multichannel Weibull fading model, where the the envelope $Z_{\ell}$ of the $\ell$ th channel $(\ell=1,2, \ldots, L$, with $L$ the number of channels) can be written as a function of the Gaussian in-phase $X_{\ell}$ and quadrature $Y_{\ell}$ elements of the multipath components, so that $Z_{\ell}=\left(X_{\ell}^{2}+Y_{\ell}^{2}\right)^{1 / \beta}$, or alternatively as a function of Rayleigh distributed random variable $(\mathrm{RV})$ as

$$
Z_{\ell}=R_{\ell}^{2 / \beta}
$$

The pdf of the Rayleigh distributed $\mathrm{RV}, R_{\ell}$, is given by [1]

$$
f_{R_{\ell}}(r)=\frac{2 r}{\Omega_{\ell}} \exp \left(-\frac{r^{2}}{\Omega_{\ell}}\right)
$$

where $E\left\langle R_{\ell}^{2}\right\rangle=\Omega_{\ell}^{2}$ and $E\langle\cdot\rangle$ denotes expectation. The corresponding cdf and the $n$th order moment are given by [1]

$$
F_{R_{\ell}}(r)=1-\exp \left(-r^{2} / \Omega_{\ell}\right)
$$

and

$$
E\left\langle R_{\ell}^{n}\right\rangle=\Omega_{\ell}^{n} \Gamma(1+n / 2)
$$

respectively, where $\Gamma(\cdot)$ is the Gamma function [16, eq. $(8.310 / 1)]$ and $n$ is a positive integer.

Using (2) and following a standard method for transformation of RVs, the pdf of $Z_{\ell}$ can be easily obtained as

$$
f_{Z_{\ell}}(r)=\frac{\beta}{\Omega_{\ell}} r^{\beta-1} \exp \left(-\frac{r^{\beta}}{\Omega_{\ell}}\right)
$$

with $E\left\langle Z_{\ell}^{\beta}\right\rangle=\Omega_{\ell}$. It is easily recognized that the above pdf follows the Weibull distribution with fading parameter $\beta$, which expresses the severity of fading $(\beta \geq 0)$. As the value of $\beta$ increases, the severity of the fading decreases, while for the special case of $\beta=2$, (5) reduces to the well-known Rayleigh pdf. Defining function $d_{\tau}=1+\tau / \beta$, where, in general, $\tau$ is a nonnegative real variable, the corresponding marginal cdf and the $n$th order moment of $Z_{\ell}$ can be expressed as

$$
F_{Z_{\ell}}(r)=1-\exp \left(-\frac{r^{\beta}}{\Omega_{\ell}}\right)
$$

and

$$
E\left\langle Z_{\ell}^{n}\right\rangle=\Omega_{\ell}^{n / \beta} \Gamma\left(d_{n}\right)
$$

respectively.

\section{A. The Bivariate Weibull Model}

The pdf, the cdf, and the $(n+m)$ th order product moment of $R_{1}$ and $R_{2}(L=2)$ are expressed as [1, eq. (6.2)]

$$
\begin{aligned}
f_{R_{1}, R_{2}}\left(r_{1}, r_{2}\right)= & \frac{4 r_{1} r_{2}}{\Omega_{1} \Omega_{2}(1-\rho)} \exp \left[-\frac{1}{1-\rho}\left(\frac{r_{1}^{2}}{\Omega_{1}}+\frac{r_{2}^{2}}{\Omega_{2}}\right)\right] \\
& \times I_{0}\left[\frac{2 \sqrt{\rho} r_{1} r_{2}}{(1-\rho) \sqrt{\Omega_{1} \Omega_{2}}}\right]
\end{aligned}
$$

(9) (see at top of this page), and

$$
\begin{aligned}
E\left\langle R_{1}^{n} R_{2}^{m}\right\rangle & =(1-\rho)^{\frac{n+m-2}{2}} \Omega_{1}^{\frac{n-1}{2}} \Omega_{2}^{\frac{m-1}{2}} \Gamma\left(\frac{1+n}{2}\right) \\
& \times \Gamma\left(\frac{1+m}{2}\right){ }_{2} F_{1}\left(\frac{1+n}{2}, \frac{1+m}{2} ; 1 ; \rho\right)
\end{aligned}
$$

respectively. In the above equations, $I_{0}(\cdot)$ is the zeroth order modified Bessel of the first kind [16, eq. (8.406/1)], $Q_{1}(\cdot, \cdot)$ is the first order Marcum's Q-function [1, eq. (4.11)], ${ }_{2} F_{1}(\cdot, \cdot ; \cdot ; \cdot)$ is the Gauss hypergeometric function [16, eq. (9.100)], and $\rho$ is the (Gaussian) correlation coefficient between $R_{1}^{2}$ and $R_{2}^{2}$ defined as

$$
\rho \triangleq \operatorname{cov}\left(R_{1}^{2}, R_{2}^{2}\right) /\left[\sqrt{\operatorname{var}\left(R_{1}^{2}\right)} \sqrt{\operatorname{var}\left(R_{2}^{2}\right)}\right] .
$$

1) Joint pdf: By applying the RVs transformation of (1) in (8), the joint pdf of the Weibull distributed envelopes RVs $Z_{1}$ and $Z_{2}$ can be obtained in closed-form as

$$
f_{Z_{1}, Z_{2}}\left(r_{1}, r_{2}\right)=\frac{\beta^{2}}{4}\left(r_{1} r_{2}\right)^{\beta / 2-1} f_{R_{1}, R_{2}}\left(r_{1}^{\beta / 2}, r_{2}^{\beta / 2}\right) \text {. }
$$

2) Correlation coefficient: With the aid of (1), the product moments of $(n+m)$ th order of $Z_{1}$ and $Z_{2}$ can be derived as

$$
E\left\langle Z_{1}^{n} Z_{2}^{m}\right\rangle=E\left\langle R_{1}^{2 n / \beta} R_{2}^{2 m / \beta}\right\rangle
$$

which using (10), yields

$$
\begin{aligned}
E\left\langle Z_{1}^{n} Z_{2}^{m}\right\rangle= & (1-\rho)^{(n+m) / \beta-1} \Omega_{1}^{n / \beta-1 / 2} \Omega_{2}^{m / \beta-1 / 2} \\
& \times \Gamma\left(\frac{1+2 m / \beta}{2}\right) \Gamma\left(\frac{1+2 n / \beta}{2}\right) \\
& \times{ }_{2} F_{1}\left(\frac{1+2 m / \beta}{2}, \frac{1+2 n / \beta}{2} ; 1 ; \rho\right) .
\end{aligned}
$$

Moreover, the correlation coefficient between $Z_{1}^{2}$ and $Z_{2}^{2}$ can be expressed as a function of the correlation coefficient between $R_{1}^{2}$ and $R_{2}^{2}$ as follows

$$
\varrho=\frac{E\left\langle Z_{1}^{2}, Z_{2}^{2}\right\rangle-E\left\langle Z_{1}^{2}\right\rangle E\left\langle Z_{2}^{2}\right\rangle}{\sqrt{E\left\langle Z_{1}^{4}\right\rangle-E^{2}\left\langle Z_{1}^{2}\right\rangle} \sqrt{E\left\langle Z_{2}^{4}\right\rangle-E^{2}\left\langle Z_{2}^{2}\right\rangle}} .
$$




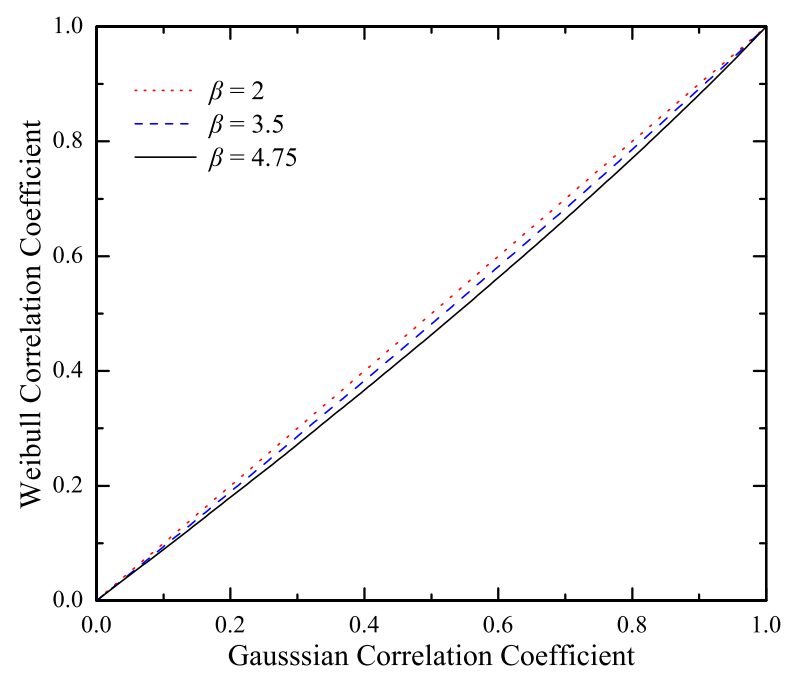

Fig. 1. Weibull Correlation coefficient, $\varrho$, as a function of the Gaussian correlation coefficient, $\rho$.

Using (7) and (14) and after some straightforward simplifications, $\varrho$ can be obtained in closed-form as

$$
\varrho=\frac{(1-\rho){ }^{d_{4}}{ }_{2} F_{1}\left(d_{2}, d_{2} ; 1 ; \rho\right)}{\Gamma\left(d_{4}\right) / \Gamma^{2}\left(d_{2}\right)-1} .
$$

In Fig. $1, \varrho$ is plotted as a function of $\rho$ for several values of $\beta$. It is clear, that $\varrho$ also ranges between zero and unity as $\rho$ does. Moreover, for a fixed value of $\rho$, as $\beta$ increases $\varrho$ decreases, while for the special cases of $\rho=0$ and $\rho=1$, $\varrho=0$ and $\varrho=1$, respectively.

3) Joint cdf: The joint cdf of $Z_{1}$ and $Z_{2}$ can be easily obtained, replacing $r_{1}$ and $r_{2}$ with $r_{1}^{\beta / 2}$ and $r_{2}^{\beta / 2}$, respectively, in (9), i.e.,

$$
F_{Z_{1}, Z_{2}}\left(r_{1}, r_{2}\right)=F_{R_{1}, R_{2}}\left(r_{1}^{\beta / 2}, r_{2}^{\beta / 2}\right) .
$$

4) Joint mgf: Rewriting the Bessel function in (12) with an infinite series representation [16, eq. (8.445)], the joint pdf of $Z_{1}$ and $Z_{2}$ can be rewritten as

$$
\begin{array}{r}
f_{Z_{1}, Z_{2}}\left(r_{1}, r_{2}\right)=\beta^{2} \exp \left[-\frac{1}{1-\rho}\left(\frac{r_{1}^{\beta}}{\Omega_{1}}+\frac{r_{2}^{\beta}}{\Omega_{2}}\right)\right] \\
\times \sum_{k=0}^{\infty} \frac{1}{(k !)^{2}} \frac{\rho^{k}}{(1-\rho)^{2 k+1}} \frac{\left(r_{1} r_{2}\right)^{-1+(k+1) \beta}}{\left(\Omega_{1} \Omega_{2}\right)^{k+1}} .
\end{array}
$$

Using the above equation, the joint mgf of $Z_{1}$ and $Z_{2}$ can be derived as $\mathcal{M}_{Z_{1}, Z_{2}}\left(s_{1}, s_{2}\right)=E\left\langle\exp \left(-s_{1} Z_{1}-s_{2} Z_{2}\right)\right\rangle$, where some integrals of the form defined as $\Upsilon(\xi, d)=$ $\int_{0}^{\infty} x^{d-1} \exp \left(-x-\xi x^{\beta}\right) d x$ appear, with $d$ and $\xi$ being arbitrary positive values. The same kind of integrals has been already analytically solved in [15], in terms of the Meijer's G-function as

$$
\Upsilon(\xi, d)=\frac{\sqrt{k / l} l^{d}}{(2 \pi)^{(k+l) / 2-1}} G_{l, k}^{k, l}\left[\begin{array}{l|l}
\xi^{k} \frac{l^{l}}{k^{k}} & \begin{array}{c}
\frac{1-d}{l}, \frac{2-d}{l}, \ldots, \frac{l-d}{l} \\
0, \frac{1}{k}, \ldots, \frac{k-1}{k}
\end{array}
\end{array}\right]
$$

with $l / k=\beta, k$ and $l$ positive integers, and $G[\cdot]$ being the Meijer's G-function [16, eq. (9.301)]. Depending upon the value of $\beta$, a set with minimum values of $k$ and $l$ can be properly chosen (e.g. for $\beta=3.5$ we have to choose $k=2$ and $l=7)$. For the special case where $\beta$ is an integer, it must be set $k=1$ and $l=\beta$. Thus, using (19), the joint mgf of $Z_{1}$ and $Z_{2}$ can be obtained as

$$
\begin{aligned}
& \mathcal{M}_{Z_{1}, Z_{2}}\left(s_{1}, s_{2}\right)=\beta^{2} \sum_{q=0}^{\infty} \frac{\rho^{q}}{(q !)^{2}(1-\rho)^{2 q+1}} \\
& \times \prod_{i=1}^{2}\left(\frac{1}{\Omega_{i} s_{i}^{\beta}}\right)^{q+1} \Upsilon\left[\frac{\left(s_{i}^{\beta} \Omega_{i}\right)^{-(q+1)}}{1-\rho},(q+1) \beta\right] .
\end{aligned}
$$

\section{B. The Multivariate Weibull Model}

Several correlation models have been proposed and used for the performance analysis of various wireless systems, corresponding to specific modulation, detection and diversity combining schemes. One of the most frequently used models is the exponential correlation model and corresponds to the scenario of multichannel reception from equispaced diversity antennas, in which the correlation between the pairs of combined signals decays as the spacing between the antennas increases.

Using the exponentially correlated Rayleigh model presented in [8], the exponentially correlated Weibull model can be derived. The multivariate pdf of identically distributed (i.d.) Rayleigh RVs, $\left\{R_{\ell}\right\}_{\ell=1}^{L}$, with exponential correlation, is given by $[8$, eq. (57)]

$$
\begin{aligned}
f_{\overrightarrow{\mathbf{R}}} & (\overrightarrow{\mathbf{r}})=\left(\frac{2}{\Omega}\right)^{L} \frac{\prod_{i=1}^{L} r_{i}}{\left(1-\rho^{2}\right)^{L-1}} \prod_{i=1}^{L-1} I_{0}\left[\frac{2 \rho r_{i} r_{i+1}}{\left(1-\rho^{2}\right) \Omega}\right] \\
& \times \exp \left\{\frac{-1}{\left(1-\rho^{2}\right) \Omega}\left[r_{1}^{2}+r_{L}^{2}+\left(1+\rho^{2}\right) \sum_{i=2}^{L-1} r_{i}^{2}\right]\right\}
\end{aligned}
$$

where $E\left\langle R_{\ell}^{2}\right\rangle=\Omega^{2}, \overrightarrow{\mathbf{r}}=\left(r_{1}, r_{2}, \ldots, r_{L}\right)$, and $\overrightarrow{\mathbf{R}}=$ $\left(R_{1}, R_{2}, \ldots, R_{L}\right)$. The correlation coefficient between $R_{i}^{2}$ and $R_{j}^{2}$ is given by $\rho_{i, j}=\rho^{|i-j|}$, when $i \neq j$, while $\rho_{i, j}=1$, when $i=j$, with $i, j=1,2, \ldots, L$.

1) Joint pdf: By applying the transformation given by (1) in (21), the joint pdf of the Weibull distributed envelopes RVs $\overrightarrow{\mathbf{Z}}=\left(Z_{1}, Z_{2}, \ldots, Z_{L}\right)$ can be obtained in closed-form as

$$
\begin{aligned}
f_{\overrightarrow{\mathbf{z}}}(\overrightarrow{\mathbf{r}})=\left(\frac{\beta}{\Omega}\right)^{L} \frac{\prod_{i=1}^{L} r_{i}^{\beta-1}}{\left(1-\rho^{2}\right)^{L-1}} \prod_{i=1}^{L-1} I_{0}\left[\frac{2 \rho\left(r_{i} r_{i+1}\right)^{\beta / 2}}{\left(1-\rho^{2}\right) \Omega}\right] \\
\quad \times \exp \left\{\frac{-1}{\Omega\left(1-\rho^{2}\right)}\left[r_{1}^{\beta}+r_{L}^{\beta}+\left(1+\rho^{2}\right) \sum_{i=2}^{L-1} r_{i}^{\beta}\right]\right\} .
\end{aligned}
$$

The correlation coefficient between $Z_{i}^{2}$ and $Z_{j}^{2}$ is given by $\varrho_{i, j}=\varrho^{|i-j|}$, when $i \neq j$, while $\varrho_{i, j}=1$, when $i=j$, with $i, j=1,2, \ldots, L$ and $\varrho$ given by (16). Substituting the Bessel function in (22), with its infinite series representation [16, eq. (8.445)] resulting in (23) (see top of the next page). 


$$
\begin{aligned}
f_{\overrightarrow{\mathbf{Z}}}(\overrightarrow{\mathbf{r}})= & \frac{(\beta / \Omega)^{L}}{\left(1-\rho^{2}\right)^{L-1}} \exp \left\{-\frac{1}{\Omega\left(1-\rho^{2}\right)}\left[r_{1}^{\beta}+r_{L}^{\beta}+\left(1+\rho^{2}\right) \sum_{j=2}^{L-1} r_{j}^{\beta}\right]\right\} \\
& \times \sum_{k_{1}, k_{2}, \ldots, k_{L-1}=0}^{\infty}\left[\frac{\rho}{\Omega\left(1-\rho^{2}\right)}\right]^{2 \sum_{i=1}^{L-1} k_{i}} \frac{r_{1}^{\left(k_{1}+1\right) \beta-1} r_{L}^{\left(k_{L-1}+1\right) \beta-1} \prod_{i=2}^{L-1} r_{i}^{\beta\left(k_{i}+k_{i-1}+1\right)-1}}{\prod_{l=1}^{L-1}\left(k_{l} !\right)^{2}}
\end{aligned}
$$

$$
\begin{aligned}
F_{\gamma_{s c}}(\gamma)= & -\exp \left[-\left(\frac{\gamma}{a \bar{\gamma}_{1}}\right)^{\beta / 2}\right] Q_{1}\left[\sqrt{\frac{2}{1-\rho}}\left(\frac{\gamma}{a \bar{\gamma}_{2}}\right)^{\beta / 4}, \sqrt{\frac{2 \rho}{1-\rho}}\left(\frac{\gamma}{a \bar{\gamma}_{1}}\right)^{\beta / 4}\right] \\
& -\exp \left[-\left(\frac{\gamma}{a \bar{\gamma}_{2}}\right)^{\beta / 2}\right]\left\{1-Q_{1}\left[\sqrt{\frac{2 \rho}{1-\rho}}\left(\frac{\gamma}{a \bar{\gamma}_{2}}\right)^{\beta / 4}, \sqrt{\frac{2}{1-\rho}}\left(\frac{\gamma}{a \bar{\gamma}_{1}}\right)^{\beta / 4}\right]\right\}
\end{aligned}
$$

2) Joint cdf: The multivariate cdf of i.d. Weibull distributed RVs, $\left\{R_{\ell}\right\}$, with exponential correlation, can be easily derived using (23) as

$$
\begin{aligned}
& F_{\overrightarrow{\mathbf{Z}}}(\overrightarrow{\mathbf{r}})=\left(1-\rho^{2}\right) \sum_{k_{1}, k_{2}, \ldots, k_{L-1}=0}^{\infty} \prod_{i=1}^{L-1}\left(\frac{\rho^{k_{i}}}{k_{i} !}\right)^{2} \\
& \times \gamma\left[k_{1}+1, \frac{r_{1}^{\beta}}{\Omega\left(1-\rho^{2}\right)}\right] \gamma\left[k_{L-1}+1, \frac{r_{L}^{\beta}}{\Omega\left(1-\rho^{2}\right)}\right] \\
& \times \frac{\prod_{j=2}^{L-1} \gamma\left[k_{j-1}+k_{j}+1, \frac{r_{j}^{\beta}\left(1+\rho^{2}\right)}{\Omega\left(1-\rho^{2}\right)}\right]}{\left(1+\rho^{2}\right)^{L-2+k_{1}+k_{L-1}+\sum_{l=2}^{L-2} 2 k_{l}}}
\end{aligned}
$$

where $\gamma(\cdot, \cdot)$ is the lower incomplete Gamma function [16, eq. (8.350/1)], which for integer values of the first argument can be further reduced to standard functions [16, eq. (8.352/1)], but this presentation is omitted due to space limitations.

\section{Outage Performance of SC Receivers}

The baseband received signal in the $\ell$ th $(\ell=1,2, \ldots, L)$ antenna of the $\mathrm{SC}$ is $\zeta_{\ell}=s Z_{\ell}+n_{\ell}$, where $s$ is the transmitted symbol of energy $E_{s}=E\left\langle|s|^{2}\right\rangle$ and $n_{\ell}$ is the additive white Gaussian noise with a single-sided power spectral density $N_{0}$. The instantaneous SNR per symbol for each diversity channel can be expressed as $\gamma_{\ell}=Z_{\ell}^{2} E_{s} / N_{0}$. The corresponding average SNR per symbol is $\bar{\gamma}_{\ell}=E\left\langle Z_{\ell}^{2}\right\rangle E_{s} / N_{0}=$ $\Gamma\left(d_{2}\right) \Omega_{\ell}^{2 / \beta} E_{s} / N_{0}$. Using the property of the Weibull distribution, that the $n$th power of a Weibull RV with parameters $\left(\beta, \Omega_{\ell}\right)$ (see (5)) gives another Weibull distributed RV with parameters $\left(\beta / n, \Omega_{\ell}\right)$, it can be easily derived that the SNR per symbol of the $\ell$ th channel is also a Weibull RV with parameters $\left(\beta / 2,\left(a \bar{\gamma}_{\ell}\right)^{\beta / 2}\right)$ and $a=1 / \Gamma\left(d_{2}\right)$. Hence, having available a formula for the fading envelopes, as those in the previous section, the corresponding expression of the SNRs per symbol can be easily derived, and thus, studying the performance of diversity receivers over correlated fading environments ${ }^{1}$.

\footnotetext{
${ }^{1}$ Using the analytical tools presented in Section II, a complete analytical performance study, including moments, error performance, etc, of several diversity receivers can be presented. However, only the outage performance of SC receivers is presented, due to space limitations.
}

\section{A. Dual-Branch SC Receivers}

The instantaneous SNR per symbol at the output of a dualbranch $(L=2)$ SC receiver will be the one with the highest value between the two branches, i.e., $\gamma_{s c}=\max \left\{\gamma_{1}, \gamma_{2}\right\}$. Using (17), the cdf of $\gamma_{s c}$ can be obtained in closed-form as in (25) (see at top of this page). Since the Marqum's Q-function is not included in the most well-known mathematical software packages, such as Mathematica and Maple, alternatively, this cdf can be expressed in the form of an infinite series representation. Hence, using (18), the joint cdf of $\gamma_{1}$ and $\gamma_{2}$ can be derived as $F_{\gamma_{1}, \gamma_{2}}\left(\gamma_{1}, \gamma_{2}\right)=\int_{0}^{\gamma_{1}} \int_{0}^{\gamma_{2}} f_{\gamma_{1}, \gamma_{2}}\left(\gamma_{1}, \gamma_{2}\right) d \gamma_{1} d \gamma_{2}$, which using [16, eq. (8.350/1) and (8.352/1)], yields

$$
\begin{aligned}
& F_{\gamma_{1}, \gamma_{2}}\left(\gamma_{1}, \gamma_{2}\right)=\sum_{k=0}^{\infty}(1-\rho) \rho^{k} \\
& \times \prod_{i=1}^{2}\left\{1-\exp \left[-\frac{1}{1-\rho}\left(\frac{\gamma_{i}}{a \bar{\gamma}_{i}}\right)^{\beta / 2}\right]\right. \\
& \left.\quad \times \sum_{m=0}^{k} \frac{1}{m !} \frac{1}{(1-\rho)^{m}}\left(\frac{\gamma_{i}}{a \bar{\gamma}_{i}}\right)^{m \beta / 2}\right\} .
\end{aligned}
$$

Using (26), the cdf of $\gamma_{s c}$ can be obtained setting $\gamma=\gamma_{1}=\gamma_{2}$ as

$$
F_{\gamma_{s c}}(\gamma)=F_{\gamma_{1}, \gamma_{2}}(\gamma, \gamma)
$$

Differentiating (27) with respect to $\gamma$, the pdf of $\gamma_{s c}$ can be also obtained as in (28) (see at top of the next page).

The outage probability, $P_{\text {out }}$, is defined as the probability that the SC output SNR falls below a given threshold, $\gamma_{t h}$, and can be obtained by replacing $\gamma$ with $\gamma_{t h}$ in (27) as

$$
P_{\text {out }}\left(\gamma_{t h}\right)=F_{\gamma_{s c}}\left(\gamma_{t h}\right) \text {. }
$$

Having numerically evaluated (29), in Fig. 2, $P_{\text {out }}$ is plotted as a function of the normalized outage threshold, $\gamma_{t h} / \bar{\gamma}_{1}$, for a dual-branch SC, with unequal input SNRs per symbol $\left(\bar{\gamma}_{2}=1.25 \bar{\gamma}_{1}\right)$, and for different values of $\beta$ and $\varrho=0.5$ (correlated). For comparison purposes, the curve for $\varrho=0$ (uncorrelated) is also included as a special case for best performance. The obtained results clearly show that $P_{\text {out }}$ degrades with an increase of the $\varrho$ and/or fading severity. 


$$
\begin{aligned}
& f_{\gamma_{s c}}(\gamma)=\frac{\beta}{2} \sum_{k=0}^{\infty} \frac{\rho^{k}}{k !(1-\rho)^{k}}\left\{\frac{\exp \left[-\left[\gamma /\left(a \bar{\gamma}_{1}\right)\right]^{\beta / 2} /(1-\rho)\right]}{\left(a \bar{\gamma}_{1}\right)^{(k+1) \beta / 2}}\left[1-\exp \left[-\frac{\left[\gamma /\left(a \bar{\gamma}_{2}\right)\right]^{\beta / 2}}{1-\rho}\right]\right] \sum_{m=0}^{k} \frac{(1-\rho)^{-m}}{m !}\right. \\
& \left.\times \frac{\gamma^{-1+(m+k+1) \beta / 2}}{\left(a \bar{\gamma}_{2}\right)^{m \beta / 2}}+\frac{\exp \left[-\left[\gamma /\left(a \bar{\gamma}_{2}\right)\right]^{\beta / 2} /(1-\rho)\right]}{\left(a \bar{\gamma}_{2}\right)^{(k+1) \beta / 2}}\left[1-\exp \left[-\frac{\left[\gamma /\left(a \bar{\gamma}_{1}\right)\right]^{\beta / 2}}{1-\rho}\right]\right] \sum_{m=0}^{k} \frac{(1-\rho)^{-m}}{m !} \frac{\gamma^{-1+(m+k+1) \beta / 2}}{\left(a \bar{\gamma}_{1}\right)^{m \beta / 2}}\right\}
\end{aligned}
$$

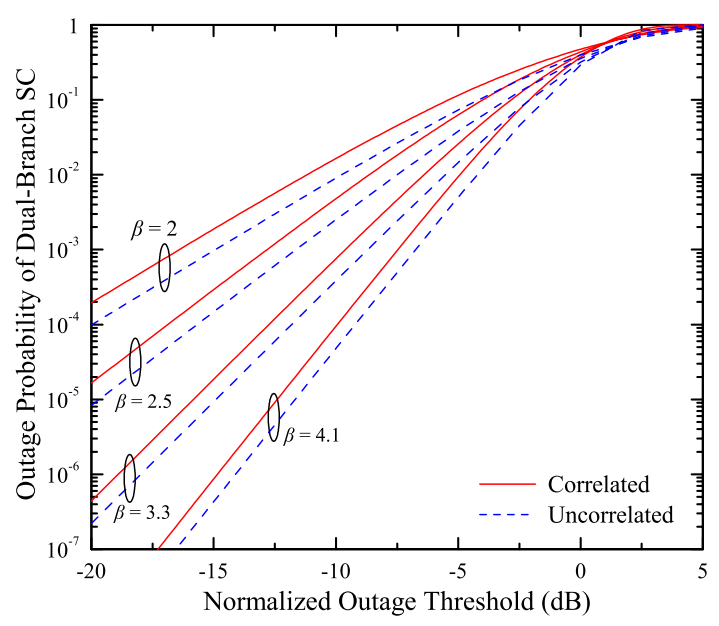

Fig. 2. Outage probability of dual-branch SC for $\bar{\gamma}_{2}=1.25 \bar{\gamma}_{1}$.

\section{B. Multi-Branch SC Receivers}

The outage probability of multi-branch SC receivers can be obtained using (24), following the procedure described in Section III, i.e., replacing $\beta$ with $\beta / 2$ and $\Omega$ with $(a \bar{\gamma})^{\beta / 2}$ $\left(\bar{\gamma}=\bar{\gamma}_{\ell}, \forall \ell\right)$, and setting $\gamma_{t h}=r_{\ell}, \forall \ell$. In Fig. 3, $P_{\text {out }}$ is plotted as a function of the normalized outage threshold, $\gamma_{t h} / \bar{\gamma}$, for a triple-branch SC, with i.d. input average SNRs per symbol, exponential correlation, and same parameters as in Fig. 2. It is observed that same findings are also observed.

\section{ACKNOWLEDGMENTS}

This work has been performed within the framework of the Satellite Network of EXcellence (SatNEx) project, a Network of Excellence (NoE) funded by European Committee (EC) under the FP6 program.

\section{REFERENCES}

[1] M. K. Simon and M.-S. Alouini, Digital Communication over Fading Channels, 1st ed. New York: Wiley, 2001.

[2] M. Nakagami, "The $m$-distribution-A general formula of intensity distribution of rapid fading," in Statistical Methods in Radio Wave Propagation. Oxford, U.K.: Pergamon Press, 1960, pp. 3-36.

[3] C. C. Tan and N. C. Beaulieu, "Infinite series representations of the bivariate Rayleigh and Nakagami- $m$ distributions," IEEE Trans. Commun., vol. 45, p. 11591161, Oct. 1997.

[4] M. K. Simon and M.-S. Alouini, "A simple integral representation of the bivariate Rayleigh distribution," IEEE Commun. Lett., vol. 2, pp. 128-130, May 1998.

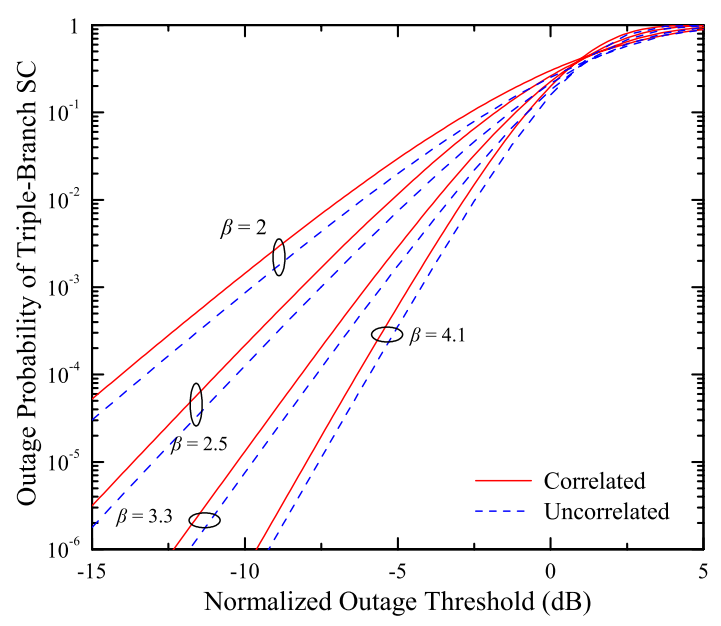

Fig. 3. Outage probability of triple-branch SC for $\varrho=0.5$ and 0 .

[5] G. K. Karagiannidis, D. A. Zogas, and S. A. Kotsopoulos, "On the multivariate Nakagami- $m$ distribution with exponential correlation," IEEE Trans. Commun., vol. 51, no. 8, pp. 1240-1244, Aug. 2003.

[6] — , "An efficient approach to multivariate Nakagami- $m$ distribution using Green's matrix approximation," IEEE Trans. Wireless Commun., vol. 2, pp. 883-889, Sept. 2003.

[7] _ "Performance analysis of triple selection diversity over exponentially correlated Nakagami- $m$ fading channels," IEEE Trans. Commun., vol. 51, pp. 1245-1248, Aug. 2003.

[8] R. K. Mallik, "On multivariate Rayleigh and exponentials distributions," IEEE Trans. Inform. Theory, vol. 49, no. 6, pp. 1499-1515, June 2003.

[9] H. Hasemi, "The indoor radio propagation channel," Proc. IEEE, vol. 81, pp. 943-968, July 1993.

[10] N. S. Adawi, et al., "Coverage prediction for mobile radio systems operating in the $800 / 900 \mathrm{MHz}$ frequency range," IEEE Trans. Veh. Technol., vol. 37, pp. 3-72, Feb. 1988.

[11] M. D. Jacoub, "The $\alpha-\mu$ distribution: A general fading distribution," in Proc. IEEE International Symposium on Personal, Indoor, and Mobile Radio Communications, Lisbon, Sept. 2002.

[12] M.-S. Alouini and M. K. Simon, "Performance of generalized selection combining over Weibull fading channels," in Proc. IEEE Vehicular Technology Conference, Atlantic City, NJ, USA, May 2001, pp. 17351739.

[13] J. Cheng, C. Tellambura, and N. C. Beaulieu, "Performance analysis of digital modulations on Weibull fading channels," in Proc. IEEE Vehicular Technology Conference, vol. 1, USA, 2003, pp. 236-240.

[14] N. C. Sagias, P. T. Mathiopoulos, and G. S. Tombras, "Selection diversity receivers in Weibull fading: Outage probability and average signal-tonoise ratio," Electron. Lett., vol. 39, no. 25, pp. 1859-1860, Dec. 2003.

[15] N. C. Sagias, G. K. Karagiannidis, D. A. Zogas, P. T. Mathiopoulos, and G. S. Tombras, "Performance analysis of dual selection diversity in correlated Weibull fading channels," IEEE Trans. Commun., vol. 52, no. 7, pp. 1063-1067, July 2004.

[16] I. S. Gradshteyn and I. M. Ryzhik, Table of Integrals, Series, and Products, 6th ed. New York: Academic, 2000. 